\title{
QUANTUM HARMONIC OSCILLATOR SONIFICATION
}

\author{
Anna Saranti Gerhard Eckel David Pirró \\ Institute of Electronic Music and Acoustics \\ University of Music and Dramatic Arts Graz \\ Inffeldg. 10/3, $8010 \mathrm{Graz}$, Austria \\ anna.saranti@student.kug.ac.at eckeleiem.at \\ pirrodiem.at
}

\begin{abstract}
This work deals with the sonification of a quantum mechanical system and the processes that occur as a result of its quantum mechanical nature and interactions with other systems. The quantum harmonic oscillator is not only regarded as a system with sonifiable characteristics but also as a storage medium for quantum information. By representing sound information quantum mechanically and storing it in the system, every process that unfolds on this level is inherited and reflected by the sound. The main profit of this approach is that the sonification can be used as a first insight for two models: a quantum mechanical system model and a quantum computation model.
\end{abstract}

\section{INTRODUCTION}

The quantum harmonic oscillator is one of the most fundamental quantum mechanical systems. It describes as in classical mechanics the motion of an object subjected to a parabolic potential [1, pp. 54-63]. As every other quantum mechanical system it is described by its Hamiltonian, which for this system is solvable with known eigenstates and eigenvalues. Any state of the system can be expressed as a superposition of its eigenstates. The quantum harmonic oscillator provides a physical realization of a quantum computer model [2, pp. 283-287] where quantum information is stored in the state of the quantum harmonic oscillator and then processed through its intristic time evolution or through coupling with the environment. The sonification choices that were adopted in this work could also be associated with these information processing operations.

At a first step sound information is stored quantum mechanically in the system's state. Letting the system evolve in time or interact with other systems affects the state and thereby the stored information. The deformation of the stored sound reflects the characteristics and properties of the system and the processes that occur. In the cases where the eigenvalues and eigenstates are affected, their sonification could also add more insight to the phenomena.

The motivation for this approach is to gain a first insight to quantum computational storage operations through sound. Quantum mechanical memory has in general different properties from the classical [2, pp. 13-17], which can be highlighted through sonification. The impact of an external disturbance to the stored quantum information is a fairly complex procedure with interdependencies that can be perceived coherently through sound. The part of the stored quantum information which is classically accessible through quantum measurement and the impact of the measurement operations in the classically retrieved part can be also acoustically represented with the use of this approach.
The best known model of a quantum mechanical memory unit is the qubit [2, pp. 13-17] which is abstract and unbounded from the properties of the physical system that realizes it. The harmonic oscillator quantum computer model bases on the features of the underlying system and therefore the representation of the quantum information is directly interconnected with the system properties.

Many other problems of quantum mechanics such as the single mode of an electromagnetic field in a one-dimensional cavity and the vibration spectra of diatomic molecules base on the quantum harmonic oscillator [1, pp. 19-32]. Thus this sonification could be a start to gain knowledge for more than one quantum mechanical system that are of the same form but also quantum mechanical systems in general because they follow the same principles.

This paper is organized as follows: The second section provides a very brief description of the system that is needed for the understanding of the sonification decisions. The third section concentrates on the sonification of the time evolution process of the quantum harmonic oscillator as a closed system, which is a derivation of the time-dependent Schrödinger equation. In the fourth section the system is subjected in two types of disturbances where the influence of the interactions with several other systems is described with the help of perturbation theory. The fifth section provides some details of the implementation whereas the sixth section presents some future plans and ideas for future works.

\section{QUANTUM HARMONIC OSCILLATOR}

\subsection{Description of the system}

Every quantum mechanical system's total energy is described by its Hamiltonian $\hat{H}$. Leaving the time evolution of the system aside and concentrating on the description of the system for a specific time point, the time-independent Schrödinger equation is [1, pp. 19-32]:

$$
\hat{H} \psi(x)=E \psi(x)
$$

where $\hat{H}$ is the Hamiltonian of the system, $\psi(x)$ the wavefunction that represents the state of the system and $E$ the eigenvalues of $\hat{H}$. The value of $|\psi(x)|^{2}$ expresses the probability density of finding the oscillating object at the position $x$ [3, pp. 54-57]. The Hamiltonian $\hat{H}$ is mathematically represented by the equation [1, pp. 54-63]:

$$
\hat{H}=K+V=\frac{\hat{p}^{2}}{2 m}+\frac{m \omega^{2}}{2} \hat{x}^{2}
$$

Where $K$ is the kinetic energy, $V$ the potential energy, $\hat{p}$ the momentum operator, $m$ the mass of the oscillating particle, $\omega$ the 
eigenfrequency and $\hat{x}$ the displacement operator. The eigenvalues that satisfy the equation (1) are quantized and represent the eigenenergies of the quantum harmonic oscillator:

$$
E_{n}=\left(n+\frac{1}{2}\right) \hbar \omega, \quad n=0,1,2 \ldots
$$

The eigenstates that satisfy the equation (1) are mathematically expressed with the help of the Hermite Polynomials $H_{n}(x)$ :

$$
H_{n}(x)=(-1)^{n} e^{x^{2}} \frac{\mathrm{d}^{n}}{\mathrm{~d} x^{n}} e^{-x^{2}}, \quad n=0,1,2 \ldots
$$

The eigenstates $\psi_{n}(x)$ which satisfy the equation (1) are weighted Hermite polynomials and represent the eigenfunctions of the quantum harmonic oscillator:

$$
\psi_{n}(x)=\left(\frac{a}{\sqrt{\pi} n ! 2^{n}}\right)^{\frac{1}{2}} H_{n}(a x) e^{-\frac{a^{2}}{2} x^{2}}, \quad a=\sqrt{\frac{m \omega}{\hbar}}
$$

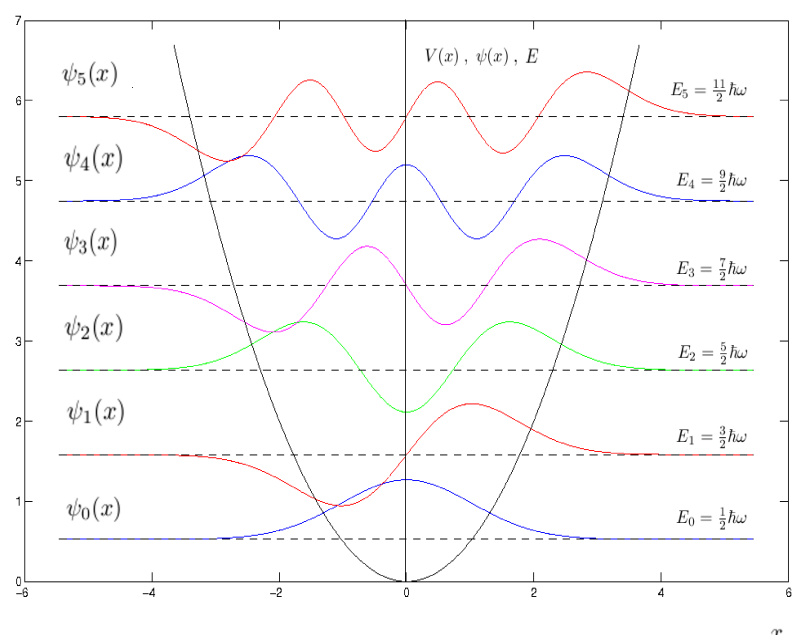

Figure 1: The first six (from 0 to 5) Eigenenergies and Eigenfucntions of the Quantum Harmonic Oscillator. The Eigenergies are depicted with equally spaced vertical lines. The corresponding Eigenfunctions are shifted on the y-axis each one with offset the particular Eigenenergy. On the same plot is also the parabolic potential $V$.

The eigenfunctions of the quantum harmonic oscillator constitute a complete and orthonormal basis. Therefore any state of the system which is represented by the wavefunction can be written as a linear combination of its eigenstates.

$$
\psi(x, t)=\sum_{n=1}^{\infty} c_{n} \psi_{n}(x)
$$

The sum of all probabilities should sum up to one. The $c_{n}$ coefficients are complex numbers that are called probability amplitudes [4] and fulfill the normalization condition:

$$
\sum_{n=1}^{\infty}\left|c_{n}\right|^{2}=1
$$

\subsection{Shapelet Basis Expansion Method}

The description of the audio signals that is realized in this work bases on their decomposition onto the eigenfuctions of the quantum harmonic oscillator thus falling to the category of a non-parametric signal expansion method [5, pp. 9-21]. The signal can be expaned as a linear combination of the basis functions $\left\{\psi_{n}(x)\right\}$. The coefficients for a signal $y$ can be obtained from the following equation:

$$
y=\sum_{n} c_{n} \psi_{n} \Rightarrow c_{n}=B^{-1} y
$$

Where $B$ is the matrix that contains the eigenfunctions $\psi_{n}(x)$ of the quantum harmonic oscillator. The $\psi_{n}(x)$ functions are called Shapelets [6], [7], because they form a perturbed version of a gaussian function as shown by the equation (5). Shapelets have a main difference to wavelets, namely the various shapes of the basis functions. The wavelet transform basis functions are the same up to a scaling factor. On the other side the shapelet basis functions are of different size and form.

The first step of the sonification procedure is to store an audio signal into the quantum harmonic oscillator using the overlap add method [8, pp. 237-238]. The signal is multiplied by a sliding window of length $N$. The successive windowed signal frames are expaned as a linear combination of the eigenfunctions of the same quantum harmonic oscillator.

The number of the eigenfunctions of the quantum harmonic oscillator is theoretically infinite but in this work only finite number of eigenfunctions are implemented depending on the needs for a good analysis and resynthesis. Throughout this work for a windowed part of a signal with number of samples $N$ the number of the coefficients $c_{n}$ used was also $N$. An extended testing of this basis for audio signal processing applications can be made in future works.

\subsection{Harmonic Oscillator Quantum Computation Model}

The computational building block of a quantum mechanical memory is the qubit whereas the memory of a quantum computer consists of several qubits [2, pp. 13-17]. As with the classical bit, the qubit is realized on a physical system. The primarly difference is that this physical system is in a level where quantum mechanical phenomena are apparent and determine the properties of the storage. A detailed description of the qubit, its properties and its differences with the classical bit are beyond the scope of this paper and are not needed for the understanding of this work.

What is essential for the comprehension of this approach is that the state of the quantum harmonic oscillator is in correspondence with the state of a quantum mechanical memory created from qubits. In the quantum harmonic oscillator model one possible physical implementation of the qubits is made in such a way that the state of the whole memory can be expanded as a linear combination of the eigenfunctions $\psi_{n}(x)$ [2, pp. 283-287]. The analogy is for every $2^{N}$ eigenstates that are used for the expansion of the signal represent the quantum information storage capability of $N$ qubits because they create an equivalent complex Hilbert space.

It is assumed that the system can be prepared in a desired state through an initialization procedure. Special attention needs to be drawn to the fact that the coefficients that are computed for the expansion of the stored audio signal not only need to fulfill the equation (8) but also the normalization condition (7). For the scope of 
this work no normalization of the coefficients to probability amplitudes is made. For a future work in which a measurement procedure is included, the consideration of the probability amplitudes normalization is crucial.

\section{CLOSED SYSTEM TIME EVOLUTION}

A quantum system that evolves without coupling to the environ- $\frac{\widehat{N}}{\underline{1}}$

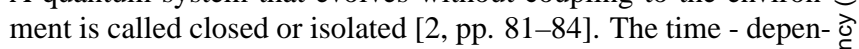
dent

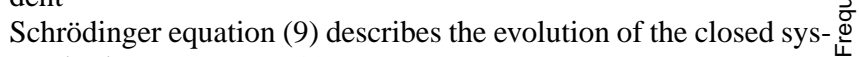
tem in time [1, pp. 19-32].

$$
i \hbar \frac{\partial \psi(x, t)}{\partial t}=\hat{H} \psi(x, t)
$$

where $\hbar$ is the Planck's constant. The time evolution is a procedure that changes the state of the system but leaves the eigenenergies and eigenfunctions unaffected. If the wavefunction of the system $\psi(x, 0)$ at time $t_{0}=0$ is desribed by the equation [2, pp. 13-17]:

$$
\psi(x, 0)=\sum_{n=1}^{\infty} c_{n}^{(0)} \psi_{n}(x)
$$

where $c_{n}^{(0)}$ are the coefficients of the input sound according to the $\psi_{n}(x)$ basis at time $t_{0}=0$, then after time $t$ each coefficient will be multiplied by a different complex exponential term:

$$
c_{n}^{(t)}=c_{n}^{(0)} e^{\frac{-i E_{n} t}{\hbar}}
$$

where $E_{n}$ is the n-th eigenenergy. The state of the system will change accordingly:

$$
\psi(x, t)=\sum_{n=1}^{\infty} c_{n}^{(t)} \psi_{n}(x)=\sum_{n=1}^{\infty} c_{n}^{(0)} e^{\frac{-i E_{n} t}{\hbar}} \psi_{n}(x)
$$

Every time that a windowed sound segment is stored in the quantum oscillator, the coefficients $c_{n}(0)$ for this window are computed with respect to the basis of the eigenfunctions $\psi_{n}(x)$. Each coefficient is then multiplied with its corresponding exponential term. The real and imaginary part of the time evolved coefficients are separately used for the resynthesis of the sound and produce two individual tracks that are merged in a stereo file. The phenomenon produces a modulation in the spectral domain which repeats itself after a specific period of time (fig. 2). The period duration $T_{n}$ of the evolution process is individual for each coefficient $c_{n}$ and is the same for the real and imaginary part:

$$
T_{n}=\frac{4 \pi}{(2 n+1) \omega}
$$

In this implementation the time variable $t$ doesn't flow continuously at each sample but for every window increases by the value of the hop size that is used in the overlap-add. With the use of a scaling factor on the time parameter the phenomenon can be heard at various speeds.

The time evolution implements a unitary transformation and therefore is the main procedure that can be used for the realization of quantum gates in this computational model [2, pp. 283-287]. With the additional use of perturbation of the eigenfunctions as described in the next chapter, information processing is achieved.

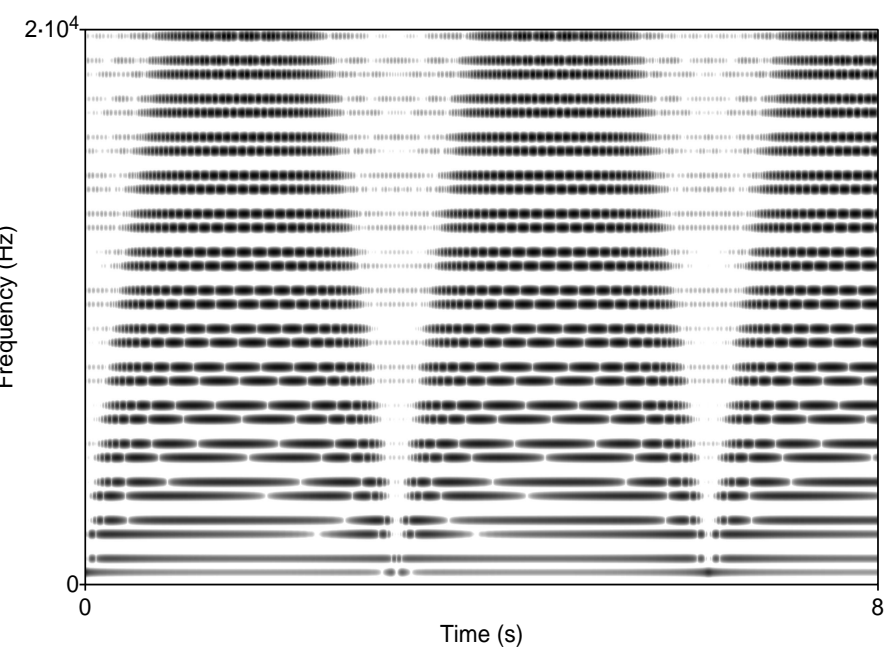

Figure 2: Spectrum of a sinusoidal of frequency $440 \mathrm{~Hz}$ expanded over the shapelet basis, then resynthesized and time-evolved. According to the time-evolution descripted by equation (12), the spectrum presents modulating frequency components that rise through the periodic change of the expansion coefficients.

\section{OPEN SYSTEM}

\subsection{Overview of Perturbation Theory}

When a quantum mechanical system has strong interactions with the environment it is called open [2, pp. 353-354]. Solving such systems i.e. finding their eigenenergies and eigenfunctions, is a complex and difficult procedure. Therefore an approximation method needs to be used. The perturbation theory is one of them and can be applied when a system with a solvable Hamiltonian $\hat{H}_{0}$ is subjected to a relatively weak disturbance $\delta \hat{H}$ in regard to the value of $\hat{H}_{0}$ [1, pp. 133]. Thus, the Hamiltonian of the overall system can be written as an addition of the exact solvable $\hat{H}_{0}$ and the disturbance $\delta \hat{H}$ :

$$
\hat{H}=\hat{H}_{0}+\delta \hat{H}
$$

The fact that this disturbance is small enough assures that there are only going to be slight changes $\delta \psi$ and $\delta E$ on the wavefunction and the energy of the system. The eigenenergies and eigenfunctions can be expressed with the help of power series:

$$
\begin{aligned}
E_{n}^{(k)} & =\frac{1}{k !} \frac{d^{k} E_{k}}{d \lambda^{k}}, \quad k=0,1,2 \ldots \\
\psi_{n}^{(k)} & =\frac{1}{k !} \frac{d^{k} \psi_{n}}{d \lambda^{k}}, \quad k=0,1,2 \ldots
\end{aligned}
$$

The perturbation $\delta \hat{H}$ corresponds to a Hamiltonian that is mathematically represented by a Hermitian matrix. In the case of the quantum harmonic oscillator with Hamiltonian $\hat{H}_{0}$ we can think of a disturbance $\delta \hat{H}$ that is a result of adding or removing some energy from the system. Throughout this work, the use of approximation approaches other than the perturbation theory are not addressed, but this could be a topic that can be further explored. 
There are two types of perturbation approaches: the timeindependent and the time-dependent. The time-independet procedure describes the system's behavior when the disturbance is constant, whereas the time-dependent deals with systems that are subjected to a time-varying disturbance.

\subsection{Time-independent or Rayleigh-Schrödinger Method}

\subsubsection{Description of the process}

The undisturbed or principal system will have an exact solution according to the time-independent Schrödinger equation $[1, \mathrm{pp}$. 134-140]:

$$
\hat{H}_{0} \psi_{n}^{(0)}(x)=E_{n}^{(0)} \psi_{n}^{(0)}(x)
$$

The zero at the superscript of $E_{n}^{(0)}$ denotes that the eigenenergies are from the undisturbed system whereas the $n$ at the subscript of the eigenenergies shows the correspondence of the $n$-th eigenenergy to the $n$-th eigenfunction. After the disturbance is applied the Hamiltonian will change by means of the equation (14) where the term $\delta \hat{H}$ is replaced by $\lambda V$ and thus the Schrödinger equation for this system will be:

$$
\hat{H} \psi_{n}(x)=\left(\hat{H}_{0}+\lambda V\right) \psi_{n}(x)=E_{n} \psi_{n}(x)
$$

Where $\hat{H}$ is the Hamiltonian, $\psi_{n}(x)$ the eigenfunctions and $E$ the eigenenergies of the of the disturbed system. The $\lambda$ term is a factor that controls the disturbance intensity and can take values with range from 0 to 1 which represent no pertubation to full perturbation accordingly. Just because the disturbance is weak, the eigenenergies $E_{n}$ of the disturbed system will not deviate very much from the eigenenergies $E^{(0)}$ of the undisturbed. The same property holds for the eigenfunctions. The power series expansion will be in accordance with the equations (15) and (16).

$$
\begin{gathered}
E_{n}=E_{n}^{(0)}+\lambda E_{n}^{(1)}+\lambda^{2} E_{n}^{(2)}+\ldots \\
\psi_{n}(x)=\psi_{n}^{(0)}(x)+\lambda \psi_{n}^{(1)}(x)+\lambda^{2} \psi_{n}^{(2)}(x)+\ldots
\end{gathered}
$$

The superscripts $0,1,2 \ldots$ denote the zero-th, first and second term of the power series. The zero superscript is the unperturbed one. The $n$ at the subscript of the eigenenergies shows the correspondence of the $n$-th eigenenergy to the $n$-th eigenfunction.

The derivation of the solution occurs by inserting the equations (19) and (20) into (18). The expression of the first order term of the eigenfunction's correction as a linear combination with respect to the orthonormal basis that is formed from the eigenfunctions $\psi_{n}^{(0)}(x)$ of the unperturbed system, leads to the first and second correction to the energy of the system:

$$
E_{n}^{(1)}=\psi_{n}^{(0)}(x)^{\dagger} V \psi_{n}^{(0)}(x), E_{n}^{(2)}=\sum_{m} \frac{\left|\psi_{m}^{(0)}(x)^{\dagger} V \psi_{n}^{(0)}(x)\right|^{2}}{E_{n}^{(0)}-E_{m}^{(0)}}
$$

The first term of the eigenfunction correction is expressed by the following equation:

$$
\psi_{n}^{(1)}(x)=\sum_{m} \frac{\psi_{m}^{(0)}(x)^{\dagger} V \psi_{n}^{(0)}(x)}{E_{n}^{(0)}-E_{m}^{(0)}} \psi_{m}^{(0)}(x)
$$

where the acute in the summation denotes that the sum is made over all $n$ eigenfunctions except the $m$. Higher terms can be obtained iteratively but are not used for the implementation to reduce the computational complexity of the implementation.

\subsubsection{Audification Choices}

For the audification of this perturbation kind various disturbance types that correspond to different Hermitian matrices $V$ were used. One example of a used perturbation corresponds to a constant electrical field with a potential that has linear dependency from the displacement $x$ which is added to the parabolic potential. The $\lambda$ factor can also be used to control how intense the development of the disturbance phenomena will be.

By applying the same disturbance type $V$ many times consecutively, a slow deformation of the shape of each of the eigenfunctions can be examined at first. The eigenenergie's values are also slightly deviating from their initial value, each one differently but consistently as a whole. Each one of the perturbation types produces a characteristic change which is clearly recognizable.

A phenomenon that occurs in every tested disturbance type, is a deformation of the eigenfrequencies and eigenvalues after the application of sufficient many consecutive time-independent perturbations. Suddenly the system starts to decompose and after a while it collapses. The eigenenergies $E_{n}$ value range grow and the implemented simulation eventually stops. The eigenfunctions $\psi_{n}(x)$ are also greatly deformed at the same time because as the eigenenergie's and eigenfunction's changes are closely linked as expressed also from the equations (21) and (22).

The alteration of the eigenfunctions can be made independently hearable by a direct mapping of the eigenfunctions in the time axis, where each channel holds one eigenfunction. Figure 3 shows the deformation of the eigenfrequencies in subsequent perturbations. One crucial point in which audification and sonification are profitable over a visual representation is the fact that the eye cannot grasp the interconnections and the consistensy of the changes of all eigenfunctions as an integrated entity.
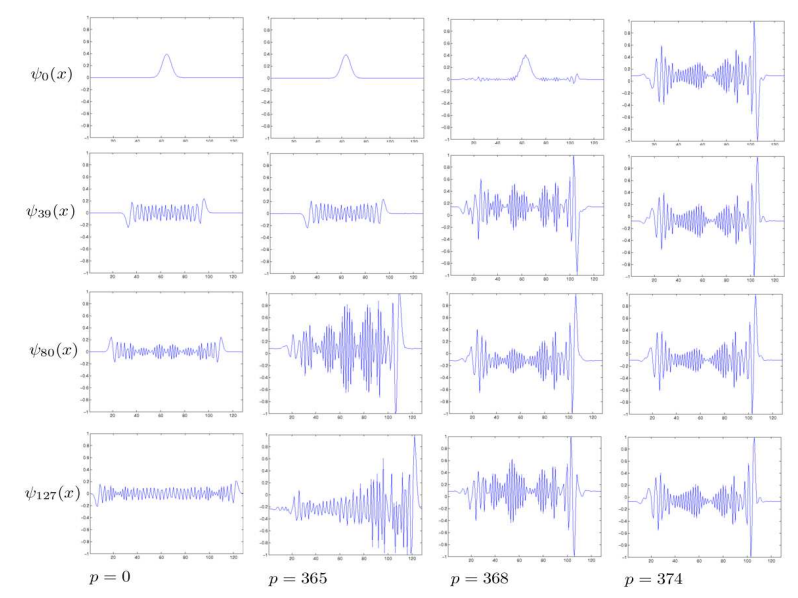

Figure 3: The deformation of the Eigenfunctions $\psi_{0}, \psi_{39}, \psi_{80}$ and $\psi_{127}$ after perturbing succesively several times. In the leftmost part of the figure the Eigenfunctions are unperturbed and at the rightmost are one step before collapsing. The value of $p$ denotes the number of time-independent perturbations that are already applied to the system. The analogy between the amplitude of the perturbed Eigenfunctions is not in direct proportion with their computed values after the desolving starts, due to normalizing conditions.

As mentioned before, the eigenfunction's transformations can 
be also made recognizable by analyzing the windowed part of the audio signal as a linear combination of the eigenbasis. In every step of the overlap-add procedure a time-independent perturbation is applied which alters the eigenfunctions in a way that they may not constitute a complete orthogonal basis anymore. Despite this fact, the coefficients are computed as if the underlying basis was orthonormal. By this means the deformation of the sound is an indication for the decompostion of the eigenfunctions and their orthonormality.

Perturbations of small intensity have no recognizable audible effects in the stored sound. The effects are starting to take place only a little before the collapsing occurs. Because of the rich content of the eigenfunction's alterations, a sonification procedure that would be more reflective of the phenomenon could be addressed.

\subsubsection{Sonification Choices}

Just because the eigenenergies of the unperturbed system are equally spaced as presented in (3), the idea of a projection of their values on the frequency plane has arised. With an appropriate scaling factor the eigenenergies can be seen as the frequencies of sinusoidals that before any perturbation create a harmonic sound. Each time the perturbation is applied the values of the frequencies of the sinusoidals are slightly changed. To make the sound effect more recognizable, the amplitude of all the sinusoidal components of the spectrum was set to the same value and then was filtered with the spectral envelope of a vowel of small duration with the help of cepstrum analysis [8, pp. 319-321].

As it can also be seen in figure 4 the first times the perturbation is applied the spectrum of the sound has a characteristic development. After a critical number of perturbations the decomposition of the system begins and an inharmonic sound is produced.

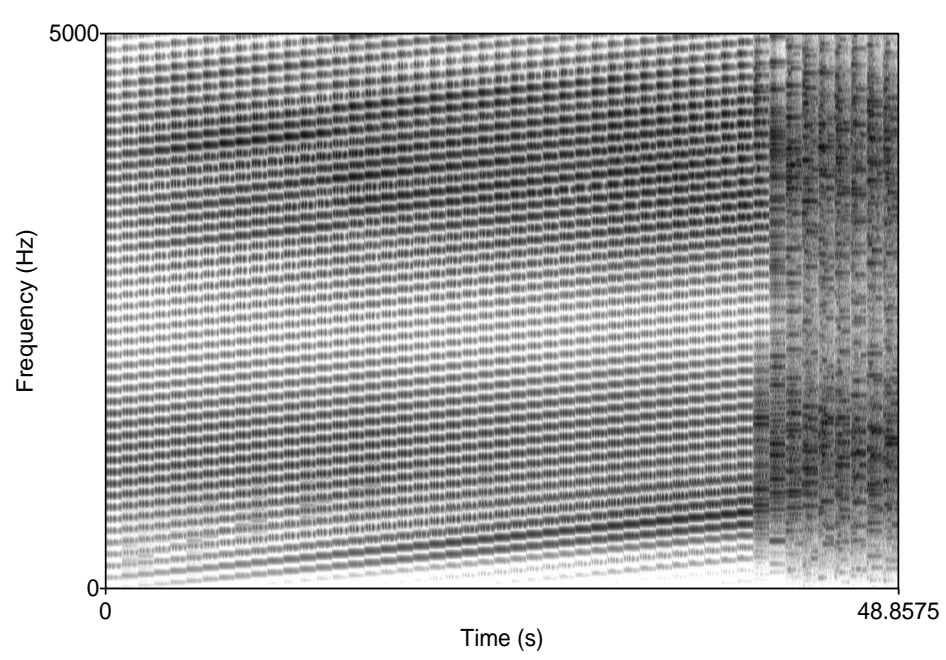

Figure 4: Spectrum of the sonified Eigenenergies after perturbing succesively several times. At each frame that is easilly seen in the figure by the vertical characteristic, the new Eigenenergies are computed. For the first perturbations the spectrum maintains a recognizable structure and after sufficiently many the dissolution of the spectral structure is apparent.

\subsection{Time-dependent or Dirac Method}

\subsubsection{Description of the process}

In this case the perturbation is denoted with the $V(t)$ operator that is assumed to be small in regard to the Hamiltonian $\hat{H}_{0}$ of the undisturbed system and the time duration of the disturbance reaction to the system is considered to be small enough. The eigenenergies and eigenfunctions of the system will also change with time. The Hamiltonian will be the addition of the unperturbed solvable and the time-dependent term [1, pp. 149-153]:

$$
\hat{H}(t)=\hat{H}_{0}+V(t)
$$

The time-dependent Schrödinger equation is in this case:

$$
i \hbar \frac{\partial \psi(x, t)}{\partial t}=\hat{H}(t) \psi(x, t)=\left(\hat{H}_{0}+V(t)\right) \psi(x, t)
$$

and cannot be solved by separating the spacial and temporal parts with the use of variable separation. That is the reason that in this case the solution cannot be implemented with the approach of the time-independent case. In analogy with the methodology of the time-independent case, the wavefunction of the system will be expanded as a linear combination of the basis of the unpertubed systm's eigenfunctions whereas the solution involves the detection of the expansion coefficients.

$$
\psi(x, t)=\sum_{m=1}^{\infty} c_{m}(t) \psi_{m}^{(0)}(x)
$$

The coefficients $c_{m}(t)$ are represented by a mathematical expression that includes both the time-evolution term that is caused from the unperturbed Hamiltonian $\hat{H}_{0}$ combined with the timedependent transformation $a_{m}(t)$ that is generated from $V(t)$ :

$$
c_{m}(t)=a_{m}(t) e^{\frac{-i E_{m}^{(0)} t}{\hbar}}
$$

The $a_{m}(t)$ terms are expanded with the help of power series. The equation (25) is solved to:

$$
\psi(x, t)=\sum_{m=1}^{\infty} a_{m}(t) e^{\frac{-i E_{m}^{(0)} t}{\hbar}} \psi_{m}^{(0)}(x)
$$

where the $a_{m}^{(1)}(t)$ is the first correction term of the $a_{m}(t)$ expansion:

$$
a_{m}^{(1)}(t)=-\frac{i}{\hbar} \int_{0}^{t} V_{n m}\left(t^{\prime}\right) e^{\frac{-i\left(E_{n}-E_{m}\right) t^{\prime}}{\hbar}} \mathrm{d} t^{\prime}
$$

and $V_{n m}(t)$ expresses the term:

$$
V_{n m}(t)=\int_{x} \psi_{m}^{(0)}(x)^{\dagger} V(t) \psi_{n}^{(0)}(x) \mathrm{d} x
$$

The further higher terms are computed iteratively but are not used in the implementation of this work due to their computational complexity.

The term $a_{m}^{(1)}(t)$ in equation (28) represents the fact that the system posesses a kind of memory. The integration is always computed from the time point where the perturbation started. Even if the disturbance stops its action the effects of the interaction are "remembered" and maintained in the system. This phenomenon is inherited to the stored sound. 


\subsubsection{Audification Choices}

The time-dependent perturbation only affects the state of the system. Therefore an insight to the influence of the disturbance can be only made through the audification of the stored sound. More specifically, for every windowed segment coefficients the first order correction term is computed and added as shown in (29). The resynthesized sound with respect to the basis of the unperturbed eigenfunctions contains the changes that are implied by the distur- $\frac{\hat{N}}{\mathrm{~N}}$ bance.

So far the type of perturbations $V(t)$ that were used could be $\frac{\bar{c}}{\overline{0}}$ decomposed as a product of a constant Hermitian matrix $V$ and a function of time $f(t)$. The $V$ term contains the spatial dependency and is in analogy with the systems that were used in the time-independent perturbation and the $f(t)$ which expresses the time dependency and contains combinations of linear, step and sinusoidal functions.

In the signals treated with a time-dependent perturbation there is always an existing component that evolves in time according to the unperturbed Hamiltonian as seen in (26) and a component that evolves under the influence of the perturbation. These two evolutions interfere with each other and create recognizable interference patterns in the spectral domain. Specially in the case where $f(t)$ is almost constant for a specific duration, a periodic component which acoustically is clearly separated from the evolution modulation appears as shown in figure 6 .

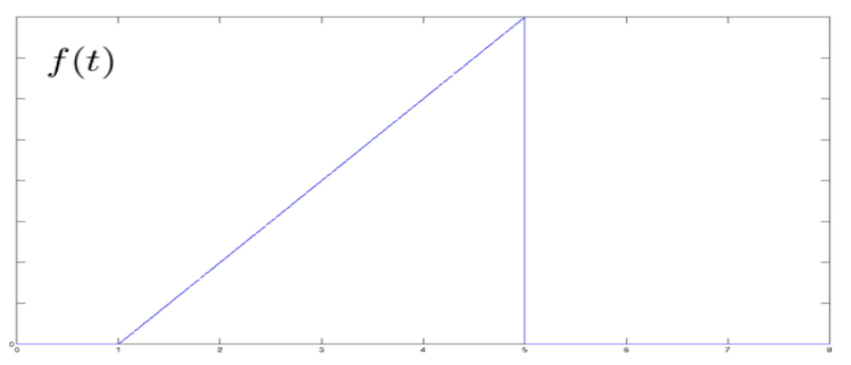

Figure 5: The time-dependency $f(t)$ of the perturbation that was used for the creation sound in figure 6

By using perturbations with different types of $V$ parts and same time dependency $f(t)$ it became apparent that the developed sounds reflect more the time dependency than the $V$ component.

\section{IMPLEMENTATION DETAILS}

Two different software packages that implement the functionality mentioned above have been programmed, one in Matlab and one in $\mathrm{C}$ for a PD external. The GNU Multiple Precision Arithmetic Library (GMP) [9] provided an efficient solution to the computation of the factorial which is the main obstacle for the implementation of the eigenfunctions $\psi_{n}(x)$ for large $n$. For the efficient computation of the externals, specially in the analysis-resynthesis stage, the CBLAS [10] and CLAPACK [11] were included.

\section{FUTURE WORK}

For the further comprehension of the system's behavior sonification decisions need to be undertaken. As seen in the eigenfunc-

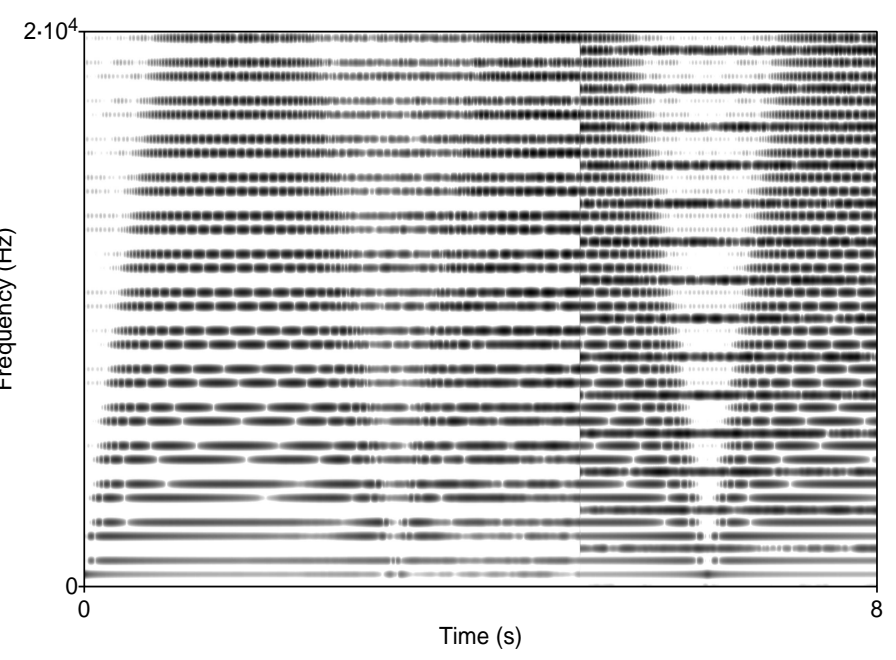

Figure 6: Spectrum of a sinusoidal with frequency $440 \mathrm{~Hz}$ when opposed to a time-dependent perturbation with $V$ a Hadamard matrix and time-dependency $f(t)$ as in the figure 5. The development of the sound has similarities with the time-evolution spectrum of the figure 2 in the beginning but the perturbation effects gradually grow. At the time point $t=5$ sec the perturbation stops but its effects remain as a constant periodical component.

tion's deformation from time-independent perturbation case, appropriate methods that include psychoacoustical principles and are stronger interconnected to the nature of some of the phenomena, should be further explored.

Apart from the pertubation method other disturbance approaches can be explored, such as the variational method [1, pp. 147-149]. This approaches are applied in the cases where the decomposi$t$ tion of the disturbed Hamiltonian cannot be made in the means of an approximative method because the system cannot be described through a small disturbance on a solvable system.

According to the correspondence principle of Bohr for relatively big values of $n$, the bahavior of the quantum harmonic oscillator should be consistent with its classical counterpart. In particular, the larger the $n$ becomes, the more we approach the classical harmonic oscillator [3, pp. 54-57]. Contrariwise, for the lowest possible value of $n$ the behavior of the oscillating object deviates the most from the classical one. Quantum mechanical phenomena obey the uncertainty principle, therefore the comparison can only be made in terms of propability densities of finding the oscillating object in a specific position $x$. A sonification scheme that will concentrate on the transition from the quantum to the classical equivalent harmonic oscillator could be one of the possible ways for the sonification of the correspondence principle.

All of the phenomena that are described until now were realized in the quantum mechanical level. How much of the stored information will be accessible in the classical level could be also explored. For the computational model to be complete a readout process of the state of the system must be included [2, pp. 283287]. The three quantum measurement schemes (General, Projective and Positive Operator Valued) will also be implemented and included in this work [2, pp. 84-93]. The audification of the measurement process, can be an approach to understand the phe- 


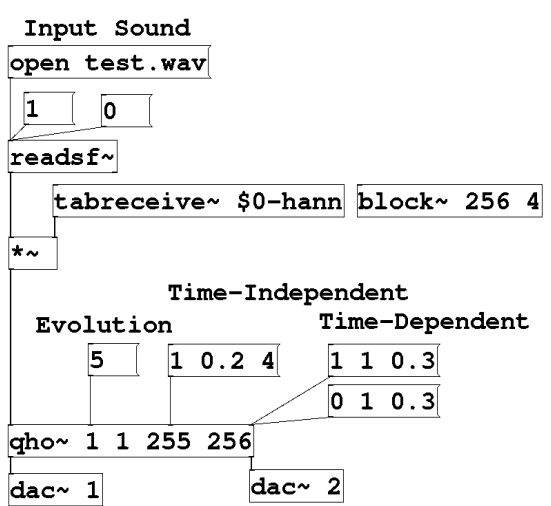

Figure 7: Pure Data External qho $\sim$ that implements the impact that the time evolution, time-independent and time-dependent perturbation procedures have to a stored test sound.

nomenon of the collapse of the wavefunction. Audio signals could be used to gain a first insight of how much of the signal information that was stored by a quantum mechanical memory is classicaly accesible.

\section{REFERENCES}

[1] F. Ehlotzky, Quantenmechanik und ihre Anwendungen, Springer-Verlag, Berlin Heidelberg, Germany, 2005.

[2] M.A. Nielsen, I.L. Chuang Quantum Computation and Quantum Information, Cambridge University Press, New York, USA, 2000.

[3] F. Schwabl, Quantenmechanik QM I Eine Einführung, Springer-Verlag, Berlin Heidelberg, Germany, Revised Edition 2005.

[4] D. Deutsch, A. Ekert, R. Lupacchini, "Machines, Logic and Quantum Physics", Bull. Symbolic Logic, vol.6, num.3, pp. 265-283, 2000

[5] M.M. Goodwin, Adaptive Signal Models - Theory, Algorithms and Audio Applications, Kluwer Academic Publishers, Boston Dordrecht London, USA, 1998

[6] A. Refregier, "Shapelets: I. A Method for Image Analysis" Mon. Not. Roy. Astron. Soc, vol. 338, no. 1, pp. 35-47, Jan. 2003.

[7] M.W. Coffey, "Properties and possibilities of quantum shapelets" J. Phys. A: Math. Gen., vol. 39, no. 4, pp. 877887 , Jan. 2006.

[8] U. Zölzer, Digital Audio Effects, John Wiley \& Sons, Chichester West Sussex, England, Reprinted 2005.

[9] GNU Multiple Precision Arithmetic Library, http://gmplib.org/, last accessed 30 January 2009

[10] CLAPACK (f2c'ed version of LAPACK), http://www.netlib.org/clapack/, last accessed 30 January 2009
[11] CBLAS Library, http://www.gnu.org/software/gsl/manual/ html_node/GSL-CBLAS-Library.html, last accessed 30 January 2009 